\title{
Categorización de la conducta verbal del cliente durante la reestructuración cognitiva
}

\author{
María Xesús Froján Parga, Ivette Vargas de la Cruz, Ana Calero Elvira y Elena Ruiz Sáncho \\ Universidad Autónoma de Madrid ${ }^{1}$
}

\section{RESUMEN}

Actualmente han surgido explicaciones sobre la técnica de reestructuración cognitiva basadas en los principios del aprendizaje asociativo, planteándose como proceso de moldeamiento verbal (Poppen, 1989). La categorización de la conducta verbal del terapeuta y del cliente es requerimiento indispensable para entender las asociaciones funcionales presentes durante este proceso. En este trabajo se propone una clasificación de la conducta verbal del cliente elaborada a partir, y como complemento, de un Sistema de categorías de la conducta verbal del terapeuta (Froján, Calero y Montaño, 2006; Froján et al., 2008; Montaño, 2008). El resultado es un sistema de codificación de 8 categorías basadas en el contenido: Proporcionar información, solicitar información, seguir instrucciones, mostrar aceptación, mostrar desacuerdo, emitir verbalizaciones de logro, emitir verbalizaciones de fracaso y emitir verbalizaciones de queja. Estos resultados constituyen el primer paso para el análisis funcional de la situación terapéutica que nos permitirá conocer los mecanismos de aprendizaje responsables del cambio clínico.

Palabras clave: Proceso terapéutico, conducta verbal, reestructuración cognitiva, análisis funcional.

\section{ABSTRACT}

Some explanations of the cognitive restructuring technique based on principles of associative learning propose that restructuring is a verbal shaping process (Poppen, 1989). Categorizing the therapist's and client's verbal behavior is an essential prerequisite for understanding the functional associations present during this process. In this paper we propose a classification of the client's verbal behavior evolving from, and complementing, the Therapist verbal behavior category system (Froján, Calero \& Montaño, 2006; Froján et al, 2008; Montaño, 2008). The result is a coding system with eight content-based categories: providing information, requesting information, following instructions, showing acceptance, showing disagreement, verbalizing achievements, verbalizing failures, and complaining. Our results constitute the first step toward a functional analysis of the therapeutic situation which will enable us to understand the learning mechanisms responsible for clinical change.

Key words: Therapeutic process, verbal behavior, cognitive restructuring technique, functional analysis 


\section{Introducción}

La técnica de reestructuración cognitiva es una de las más utilizadas en el ámbito clínico pero a pesar de ello, la explicación de su funcionamiento así como el aprendizaje de su utilización siguen siendo cuestiones confusas. Hay muchos aspectos controvertidos, desde la definición de idea irracional, defendida por Ellis (1962) como clave para entender la técnica y criticada por muy diversos autores por tautológica (Haaga y Davison, 1993; Pérez-Álvarez, 1996; Salzinger, 1992) hasta las explicaciones existentes sobre su funcionamiento, que se basan en constructos con poco significado conductual (como "error lógico" o "esquema disfuncional") y, sobre todo, no consideran las posibles funcionalidades de las cogniciones desadaptativas.

Por otra parte, no existen guías sistemáticas para su implementación y las numerosas publicaciones al respecto se centran mayoritariamente en su efectividad para el tratamiento de diferentes problemas psicológicos. Así, aunque existen numerosas aportaciones teóricas sobre la técnica y múltiples estudios sobre la eficacia de las técnicas cognitivas, nos encontramos ante un vacío de estudios empíricos sobre los procesos de aprendizaje subyacentes y muchas preguntas sin resolver sobre su indudable efectividad.

En la actualidad comienzan a desarrollarse explicaciones alternativas de la técnica; en esta línea, lo que se ha denominado cambio en las creencias irracionales o en los esquemas cognitivos se puede entender como un cambio en las verbalizaciones de la persona, desde unas menos adaptativas a otras más adaptativas que dan pie al cambio del comportamiento manifiesto. A este respecto, destacamos los trabajos de Luciano, Barnes-Holmes y Barnes-Holmes (2002) y Luciano, Herruzo y Barnes-Holmes (2001), cuyas conclusiones señalan el papel de las contingencias verbales en la creación de las correspondencias decir-hacer. Algunos autores afirman que estas correspondencias pueden modificar otras conductas hasta tal punto que podría resultar más fácil cambiar el comportamiento humano moldeando lo que se dice sobre lo que se hace que moldeando directamente lo que se hace (Catania, 1998).

Poppen (1989) y Martin y Pear (2007), siguiendo la misma línea, postulan que la reestructuración es un procedimiento para modificar las reglas erróneas que la persona se formula a sí misma. Poppen propone el debate como un procedimiento verbal en el que se dan procesos de moldeamiento y reforzamiento a través de los cuales el terapeuta consigue cambiar la regla que guía la conducta del cliente. Del mismo modo, ocurren también procesos de castigo, dado que las intervenciones del terapeuta pueden tener la función de castigo para el cliente cuando defiende sus creencias. El terapeuta enseña al cliente a discriminar y realizar afirmaciones individuales de eventos que ocurren, diferenciándolas de las formulaciones simplistas que abusan de los adverbios temporales "siempre" y "nunca"; es decir, se le provee de nuevas reglas para hacer análisis más finos de las contingencias en lugar de las antiguas sobregeneralizaciones que constituían sus reglas. Más allá de las controversias en torno a lo que se ha denominado conducta gobernada por reglas (véase, por ejemplo, 
Tabla 1

Categorías incluidas en el Sistema de Categorización de la Conducta Verbal del Terapeuta (SISC-CVT)

\begin{tabular}{|c|c|}
\hline Categoría & Descripción breve \\
\hline Función discriminativa & $\begin{array}{l}\text { Verbalización del terapeuta que da pie a una conducta del cliente (verbal o no) que va se- } \\
\text { guida de reforzamiento o castigo. (Categoría evento) }\end{array}$ \\
\hline Función evocadora & $\begin{array}{l}\text { Verbalización del terapeuta que da lugar a una respuesta emocional manifiesta en el cliente } \\
\text { acompañada de verbalización o no. (Categoría evento) }\end{array}$ \\
\hline Función de refuerzo & $\begin{array}{l}\text { Verbalización del terapeuta que muestra aprobación, acuerdo y/o aceptación, de la con- } \\
\text { ducta emitida por el cliente. (Categoría evento) }\end{array}$ \\
\hline Función de castigo & $\begin{array}{l}\text { Verbalización del terapeuta que muestra desaprobación, rechazo y/o no aceptación de la } \\
\text { conducta emitida por el cliente y/o que interrumpe ésta aunque sin presentar ningún matiz } \\
\text { que indique aprobación, acuerdo o aceptación. (Categoría evento). }\end{array}$ \\
\hline Función informativa & $\begin{array}{l}\text { Verbalización del terapeuta que transmite un conocimiento técnico o clínico a una persona } \\
\text { no experta. (Categoría estado) }\end{array}$ \\
\hline Función instruccional & $\begin{array}{l}\text { Verbalización del terapeuta encaminada a fomentar la aparición de una conducta futura del } \\
\text { cliente fuera del contexto clínico. No se han de mencionar las consecuencias explícitamente } \\
\text { pero sí han de describirse los pasos de la actuación que se trata de favorecer. (Categoría } \\
\text { estado) }\end{array}$ \\
\hline Función motivacional & $\begin{array}{l}\text { Verbalización del terapeuta que anticipa los efectos positivos o negativos que la actuación } \\
\text { del cliente (mencionada dicha actuación o no de forma explícita en la verbalización del } \\
\text { terapeuta) tendrá, está teniendo o ha tenido sobre el cambio clínico. (Categoría estado) }\end{array}$ \\
\hline Otras & $\begin{array}{l}\text { Cualquier verbalización del terapeuta que no se pueda incluir dentro de las categorías an- } \\
\text { teriores. (Categoría evento) }\end{array}$ \\
\hline
\end{tabular}

Schlinger, 1990) y sin asumir este término como explicativo de la conducta sino como descriptivo, consideramos que esta perspectiva, en términos de moldeamiento, es la adecuada para analizar la técnica de debate.

En este contexto, nuestro grupo de investigación ha iniciado un estudio de lo que sucede en terapia desde dos supuesto teóricos primordiales: (1) la conceptualización de la interacción terapeuta-cliente como un proceso de discriminación y reforzamiento a través del cual el terapeuta moldea gradualmente la conducta del cliente durante el proceso terapéutico (Callaghan, Naugle y Follette, 1996); y (2) el estudio del lenguaje observado en la clínica desde una perspectiva analítico-funcional (Hayes y Wilson, 1994; Kohlenberg y Tsai, 1991 Wilson y Blackledge, 2000). En este marco, se considera que el lenguaje, durante la sesión clínica, tiene el estatus de conducta relevante clínicamente; partiendo de estas premisas, hemos diseñado un sistema de categorías de la conducta verbal del terapeuta (Froján, Calero, y Montaño, 2006; Froján et al., 2008; Montaño, 2008) y ahora, en este trabajo, pretendemos presentar uno del cliente aplicado a la reestructuración cognitiva.

El desarrollo del Sistema de Categorías de la Conducta Verbal del Terapeuta (SISC-CVT, ver Tabla 1) se fundamenta en las operaciones básicas de conducta propuestas por Catania (Catania, 1992; Pérez-Álvarez, 1996) y en una metodología observacional de análisis de la conducta verbal del terapeuta durante la sesión clínica. La fiabilidad interjueces de dicho sistema alcanzó un índice Kappa de .74, que 
indica un nivel muy aceptable de precisión de los observadores para un sistema con 8 categorías en el que la variabilidad de las probabilidades simples de las categorías es alta (Bakeman, Quera, McArthur y Robinson, 1997).

En cuanto a la conducta verbal del cliente, no ha sido abordada desde una perspectiva analítico-funcional y las categorías de la mayoría de los estudios se han construido de acuerdo a otros aspectos, de manera que podemos considerar dos tipos principales: 1) los sistemas de categorías basados en el contenido y 2) los sistemas basados en la respuesta adaptativa. Dentro de los primeros cabe señalar la Taxonomy of verbal response modes (VRM) de Stiles (1987), que consta de 8 modos: manifestación, exposición, guía, confirmación, pregunta, señalización, interpretación y reflejo. Se registran los actos de habla de manera literal (forma) y a nivel pragmático (intención). En segundo lugar, el Client Verbal Response Category System de Hill et al. (1981) con sus 9 categorías nominales y mutuamente excluyentes para juzgar los modos de respuesta verbal del cliente: respuestas simples, peticiones, descripción, experiencias, insight, discusión de planes, discusión de la relación entre terapeuta y cliente, silencio y otras. Otros autores han desarrollado sistemas de categorías basados en el contenido, sin embargo se centran o incluyen aspectos diferentes a los que pretendemos estudiar, como por ejemplo: la implicación en la terapia (Klein, Mathieu-Coughlan y Kiesler, 1986); emoción (Canfield, Walker y Brown, 1991; Sexton, Littauer, Sexton y Tommeras, 2005); autorreferencias al afecto (Highlen y Baccus, 1977; Lavelle ,1977); orientación en el tiempo (Barna- bei, Cormier y Nye, 1974; Lavelle ,1977); incertidumbre, insight, tipo de experiencias (Lonborg et al., 1991); categorías temporales, direccionales y experienciales (Meier y Boivin, 1986) o categorías exclusivas para la terapia centrada en el cliente y para la terapia racional-emotiva (Martin, Martin y Slemon, 1987).

Dentro de los sistemas de categorías que tienen en consideración la respuesta adaptativa destacan dos sistemas: uno, formulado por Callaghan, Summers y Weidman (2003), quienes utilizaron el Functional Analytic Psychotherapy Rating Scale para describir categorías del cliente como comportamientos específicos de la FAP dentro y fuera de la sesión terapéutica, y, otro, desarrollado por Karpiak y Benjamin (2004), quienes configuraron su sistema de categorías de acuerdo a la respuesta adaptativa, según la variable contenido (interpersonal o relacionada con los síntomas) y la variable adaptación (adaptativo-no adaptativo-neutro).

Estas propuestas, a pesar de su indudable interés, no son las mejores para alcanzar nuestro objetivo, debido a que, o bien no toman en cuenta el cambio progresivo hacia una respuesta cada vez más adaptativa o lo hacen desde una perspectiva diseñada específicamente para la FAP que impide aplicarlos a otro tipo de intervenciones. Con el objetivo de superar estos inconvenientes, en este trabajo se presenta una primera propuesta de un sistema de categorización de la conducta verbal del cliente durante el desarrollo de la reestructuración cognitiva, considerando que tal conducta siempre tiene función de respuesta de la conducta del terapeuta y que 
éste puede ir moldeando progresivamente las respuestas del cliente, desde las menos a las más adaptativas.

\section{Método \\ Participantes}

Se utilizaron las grabaciones de cuatro sesiones de tratamiento de una hora de duración de tres clientes diferentes. La terapeuta que participó en el estudio, de orientación cognitivo-conductual, tenía 15 años de experiencia clínica y procedía de un gabinete psicológico privado, el Instituto Terapéutico de Madrid (ITEMA). En todos los casos la intervención psicológica llevada a cabo fue individual y con población adulta. Las grabaciones fueron realizadas con el consentimiento informado de terapeutas, clientes y la directora del centro, garantizándose en todo momento la confidencialidad y privacidad de los datos. Este procedimiento superó todos los requisitos exigidos por el Comité de Ética de la Investigación de la Universidad Autónoma de Madrid.

\section{Instrumentos}

Los casos fueron grabados utilizando un circuito cerrado de vídeo, cuyas cámaras se encontraban semiocultas. El vídeo fue grabado en formato DVD y transformado posteriormente a MPEG-2, el formato requerido por la herramienta informática de observación segundo a segundo: el software The Observer XT, comercializado por Noldus Information Technology.
Procedimiento para la creación del sistema de

$$
\text { categorías }
$$

El proceso de creación del sistema de categorías siguió las siguientes fases:

Establecimiento de la unidad de categorización: se decidió registrar la conducta verbal manifiesta emitida por el cliente durante cada uno de los fragmentos de reestructuración. El comportamiento no verbal del cliente no fue objeto de análisis, aunque se atendió al tono para entender el sentido de algunas frases que, en función de éste, podrían modificar su significado notablemente (por ejemplo, las verbalizaciones irónicas).

Elección del procedimiento de observación y registro: tres observadores independientes analizaron de manera informal los fragmentos de las sesiones clínicas y se decidió trabajar en la observación directa y registro manual de éstas para analizar la conducta verbal del cliente durante la interacción. La herramienta informática The Observer XT se utilizarían en la segunda fase de concreción del sistema de categorías.

Observación informal de la conducta: se realizaron las transcripciones literales de las sesiones y como resultado de esta tarea, se definieron las primeras categorías del cliente. Posteriormente, se procedió a la categorización no sistemática de los fragmentos grabados de restructuración y al registro informal de la ocurrencia de aquellos comportamientos verbales de los clientes que podrían incluirse en alguna de las categorías previamente formuladas. A medida que los tres jueces realizaban sus registros, éstos se comparaban para identificar y discutir los desacuerdos existentes con el fin 
Tabla 2

Categorías incluidas en el Sistema de Categorización de la Conducta Verbal del Cliente para la Reestructuración cognitiva (SISC-CVC-RC)

\begin{tabular}{|c|c|}
\hline Categoría & Descripción breve \\
\hline Proporcionar información & $\begin{array}{l}\text { Verbalización a través de la cual el cliente trata de proporcionar al terapeuta información } \\
\text { relevante para la evaluación y/o el tratamiento. }\end{array}$ \\
\hline Solicitar información & Pregunta, comentario y/o petición de información por parte del cliente hacia el terapeuta. \\
\hline Seguir instrucciones & $\begin{array}{l}\text { Conducta verbal que implica seguimiento total o parcial de instrucciones presentadas } \\
\text { inmediatamente antes por el terapeuta o verbalizaciones que aluden al cumplimiento (futuro o } \\
\text { pasado) de instrucciones fuera de sesión. }\end{array}$ \\
\hline Mostrar aceptación & $\begin{array}{l}\text { Verbalizaciones que muestren acuerdo, aceptación y/o admiración ante verbalizaciones } \\
\text { emitidas por el terapeuta. }\end{array}$ \\
\hline Mostrar desacuerdo & $\begin{array}{l}\text { Verbalizaciones que indican desacuerdo, desaprobación y/o rechazo ante verbalizaciones } \\
\text { emitidas por el terapeuta. }\end{array}$ \\
\hline $\begin{array}{l}\text { Emitir verbalizaciones } \\
\text { de logro }\end{array}$ & $\begin{array}{l}\text { Verbalizaciones del cliente que señalan la consecución de algún objetivo terapéutico o la } \\
\text { anticipación de la consecución del mismo. }\end{array}$ \\
\hline $\begin{array}{l}\text { Emitir verbalizaciones } \\
\text { de fracaso }\end{array}$ & $\begin{array}{l}\text { Verbalizaciones del cliente que señalan la no consecución de algún objetivo terapéutico o la } \\
\text { anticipación de la no consecución del mismo. }\end{array}$ \\
\hline $\begin{array}{l}\text { Emitir verbalizaciones } \\
\text { de queja }\end{array}$ & $\begin{array}{l}\text { Verbalizaciones del cliente que hacen referencia a su propio padecimiento debido a sus } \\
\text { conductas problema. }\end{array}$ \\
\hline
\end{tabular}

de especificar y depurar los criterios de categorización.

Observación sistemática del comportamiento: propuesta del sistema de codificación. En esta etapa de la investigación se inició el registro sistemático de la conducta verbal de los clientes con la ayuda del programa informático The Observer XT. Dos observadores llevaron a cabo la observación y registro independiente de las grabaciones. Tras la observación de cada sesión por parte de los jueces se comparaban los registros y se discutían los desacuerdos encontrados hasta llegar a establecer criterios consensuados que facilitaran la categorización de la conducta verbal de los clientes estudiados.

\section{Resultados}

Sistema de categorías

El proceso descrito concluyó con la especificación de ocho categorías para clasificar la conducta verbal del cliente (Tabla 2), basadas en el contenido ya que siempre se considera una única posible funcionalidad: repuesta ante las verbalizaciones del terapeuta.

\section{Discusión}

Las aportaciones que se hacen en este trabajo deben entenderse a la luz de los objetivos últimos de nuestra línea de investigación y nuestra conceptualización de la reestructuración cognitiva como un proceso de discriminación y moldeamiento en la interacción entre terapeuta y cliente en sesión. En primer lugar, las categorías de la conducta verbal del cliente que se presentan como resultado de este primer acer- 
camiento son específicas de la reestructuración cognitiva. En este sentido, permiten clasificar la conducta verbal del cliente de manera precisa y relacionada con lo que se observa durante la aplicación de esta técnica, lo que aporta un nivel de especificidad mayor que si se hubiera empleado un sistema de categorías de la conducta del cliente más general. En segundo lugar, se trata de un sistema adaptado a $-\mathrm{y}$ construido a partir de- un sistema de categorización de la conducta verbal del terapeuta que ha mostrado en diversos estudios su alto nivel de fiabilidad (Froján, Calero, y Montaño, 2006; Froján et al., 2008; Montaño, 2008). Así, se trata de dos sistemas complementarios que en un futuro permitirán estudiar la interacción terapéutica o bien la conducta de ambos interactuantes (psicólogo-cliente) de forma independiente. La categorización de la conducta verbal del cliente con uno de los sistemas de categorías ya existentes hubiera sido posible, pero no nos permitiría estudiar la interacción como un proceso de aprendizaje y nuestro objetivo último es conocer las relaciones funcionales entre estímulos verbales que se van modificando a lo largo del proceso clínico y pueden ser responsables del cambio psicológico. En este momento las categorías propuestas son morfológicas, característica que comparten con las categorías de aquellos sistemas ya citados basados en contenidos, pero en un futuro estudiaremos la función de respuesta -operante o respondente- de nuestras categorías y, por ello, se ha considerado de utilidad partir de una clasificación 'funcional' de la conducta verbal del terapeuta.

En base en: (1) el análisis de la sucesión de verbalizaciones que el cliente establece con respecto a las del terapeuta a lo largo de los debates, (2) los momentos en los que el terapeuta cambia su estrategia de debate o su objetivo conforme avanza el proceso de la reestructuración (las verbalizaciones establecidas como objetivo de debate son progresivamente más cercanas al objetivo final) y (3) las secuencias E-R-C identificadas en el análisis, se podrían proponer como hipótesis a comprobar en futuras investigaciones que el cambio de las verbalizaciones del cliente, a lo largo de la reeestructuración cognitiva, se podría explicar por un procedimiento de moldeamiento desde verbalizaciones del cliente poco adaptativas a otras más adaptativas. Para estudiar el acercamiento paulatino hacia un comportamiento más adaptativo será importante tomar en cuenta la distinción entre verbalizaciones adaptativas y no adaptativas El proceso de validación deberá completarse con nuevos estudios en los que se confronte nuestro sistema de categorías con otros códigos observacionales similares.

A modo de conclusión, este trabajo es un punto de partida para la clarificación de por qué y cómo ocurre el cambio terapéutico al aplicar la reestructuración cognitiva. Nuestro objetivo último es estudiar la interacción entre terapeuta y cliente mediante el estudio detallado de los debates. Nos centraremos en el componente verbal de la interacción terapeuta-cliente, considerando el lenguaje como conducta operante, así como en las verbalizaciones/cogniciones del cliente que el terapeuta establece como objetivo de cambio, estudiando los posibles cambios que se den en la aplicación de la técnica según avanzan las 
sesiones, esta tarea que sin duda, hará posible una mejor comprensión de lo que sucede en el campo terapéutico y, como consecuencia, una mejor efectividad terapéutica.

\section{Bibliografía}

Bakeman, R., Quera, V., McArthur, D., \& Robinson, B.F. (1997). Detecting sequential patterns and determining their reliability with fallible observers. Psychological Methods, 2, 357-370.

Barnabei, F., Cormier, W. H., \& Nye, S. (1974). Determining the effects of three counselor verbal responses on client verbal behavior. Journal of Counselling Psychology, 21, 355-359.

Callaghan, G.. M., Naugle, A. E., \& Follette, W. C. (1996). Useful constructions of the clienttherapist relationship. Psychotherapy, 33, 381-390.

Callaghan, G. M., Summers, C. J., \& Weidman, M. (2003). The treatment of histrionic and narcissistic personality disorder behaviors: A single-subject demonstration of clinical effectiveness using Functional Analytic Psychotherapy. Journal of Contemporary Psychotherapy, 33, 321-339.

Canfield, M. L, Walker, W. R., \& Brown, L. G. (1991). Contingency Interaction Analysis in Psychotherapy. Journal of Consulting and Clinical Psychology, 59, 58-66.

Catania, A. C. (1992). Learning. Nueva Jersey, Estados Unidos: Prentice Hall.

Catania, A. C. (1998). The taxonomy of verbal behaviour. En K.A. Lattal \& M. Perone (Eds.), Handbook of research methods in human operant behavior (pp. 405-433). Nueva York: Plenum Press.

Ellis, A. (1962). Reason and Emotion in Psychotherapy. New York: Litle Stuart.

Froján, M. X., Calero, A. \& Montaño, M. (2006). ¿Por qué la gente cambia en terapia? Un estudio preliminar. Psicothema, 18, 4, 797803.
Froján, M. X., Montaño, M., Calero, A., García, A., Garzón, A. \& Ruiz, E. (2008). Sistema de categorización de la conducta verbal del terapeuta. Psicothema, 20, 603-609.

Haaga, D. A., \& Davison, G. C. (1993). An appraisal of racional-emotive therapy. Journal of Consulting and Clinical Psychology, 61, 215220.

Hayes, S. C., \& Wilson, K. G. (1994). Acceptance and commitment therapy: Altering the verbal support for experiential avoidance. The Behavior Analyst, 17, 289-303.

Highlen, P. S., \& Baccus, G.. K. (1977). Effect of the reflection of feeling and probe on client self- referenced affect. Journal of Counseling Psychology, 24, 440-443.

Hill, C. E., Greenwald, C., Reed, K. G., Charles, D., O'Farrell, M. K., \& Carter, J. A. (1981). Manual for counselor and client verbal response category systems. Columbus, OH, Estados Unidos: Marathon Consulting and Press.

Karpiak, C. P., \& Benjamin, L. S. (2004). Therapist affirmation and the process and outcome of psychotherapy: Two sequential analytic studies. Journal of Clinical Psychology, 60, 659-676.

Klein, M. H., Mathieu-Coughlan, P., \& Kiesler, D. J. (1986). The Experiencing Scales. En L. Greenberg \& W. Pinsof (Eds.), The Psychotherapeutic Process. New York: Guilford.

Kohlenberg, R. J., \& Tsai, M. (1991). Functional analytic psychotherapy. Creating intense and curative therapeutic relationships. Nueva York: Plenum Press.

Lavelle, J. J. (1977). Comparing the effects of an affective and a behavioral counsellor style on client interview behaviour. Journal of Counseling Psychology, 24, 173-177.

Lonborg, S. D., Daniels, J. A., Hammond, S. G., Houghton-Wenger, B., \& Brace, L. J. (1991). Counselor and Client Verbal Response Mode Changes During Initial Counselling Sessions. Journal of Counseling Psychology, $38,394-400$. 
Luciano, M. C., Barnes-Holmes, Y., \& BarnesHolmes, D. (2002). Establishing reports of saying and doing and discriminations of say-do relations. Research in Developmental Disabilities, 23, 406-421.

Luciano, M. C., Herruzo, J., \& Barnes-Holmes, D. (2001). Generalization of say-do correspondance. The psychological Record, 51, 111130.

Martin, G., \& Pear, J. (2007). Behavior Modification: What It Is and How to Do It. Nueva Jersey, Estados Unidos: Prentice Hall.

Martin, J., Martin, W., \& Slemon, A. G. (1987). Cognitive mediation in person-centered and rational-emotive therapy. Journal of Counseling Psychology, 34, 251-260.

Meier, A., \& Boivin, M. (1986). Client Verbal Response Category System: Preliminary data. Journal of Consulting and Clinical Psychology, 54, 877-879.

Montaño, M. (2008). Estudio observacional de la conducta verbal del psicólogo para el análisis del proceso terapéutico. Tesis doctoral no publicada, Universidad Autónoma de Madrid, Madrid, España.

Pérez-Álvarez, M. (1996). Tratamientos psicológicos. Madrid: Universitas.

Poppen, R. L. (1989). Some clinical implications of rule-governed behavior. En S. C. Hayes (Ed.), Rule-governed behavior. Cognition, contingencies and instructional control (pp. 325-357). Nueva York: Plenum Press.

Salzinger, K. (1992). Cognitive therapy: A misunderstanding of B. F. Skinner. Journal of Behaviour Therapy and Experimental Psychiatry, 23, 3-8.

Schlinger, H. D. (1990). A reply to behavior analysts writing about rules and rule-governed behavior. The Analysis of Verbal Behavior, 8, 77-82.

Sexton, H., Littauer, H., Sexton, A., \& Tommeras, E. (2005). Building an alliance: Early therapy process and the client-therapist connection. Psychotherapy Research, 15, 103-116.
Stiles, W. B. (1987). Verbal response modes as intersubjective categories. En R. L. Russell (Ed.), Language in psychotherapy: Strategies of discovery (pp. 131-170). Nueva York: Plenum Press.

Wilson, K. G., \& Blackledge, J. T. (2000). Recent developments in the behavioral analysis of language: making sense of clinical phenomena. En M. J. Dougher (Ed.), Clinical behavior analysis (pp. 27-46). Reno, NV, Estados Unidos: Context Press. 
